\title{
NONLINEAR LASER CHEMISTRY OF MALEIC ACID
}

\author{
D. LEUPOLD, J. EHLERT, † S. OBERLÄNDER, $†$ E. KLOSE, \\ S. MORY and G. WINKELMANN $\ddagger$
}

Academy of Sciences of the GDR, Central Institute of Optics and Spectroscopy, Rudower Chaussee, GDR-1199 Berlin

(Received 15 May, 1988; in final form 17 June, 1988)

\begin{abstract}
With reference to recent laser investigations of excited state reactions of maleic acid in Letokhov's group, relevant excited state constants were determined by means of a physico-mathematical methods package of nonlinear absorption and the excited state populations were calculated for the experimental conditions. Based on this, a change of the assignment of the found reactions to excited states is suggested in the following manner: dimerization in $T_{1}$ and maleic acid formation in a higher excited triplet.

KEY WORDS: Excited state reactions, nonlinear absorption, physico-mathematical methods package, maleic acid, dimerization.
\end{abstract}

\section{INTRODUCTION; AIM OF PRESENT WORK}

The laser photochemistry of aqueous solutions of maleic acid ${ }^{1-4}$ is one of the most impressive examples of the realization of Porters prediction ${ }^{5}$ on wide possibilities of chemistry in excited molecular electronic states. Besides the well-known isomerization in the lowest triplet level ${ }^{6}$ (cf. reaction I in Figure 1) two other reactions in excited states were found by Letokhov et al. ${ }^{1}$ in high-intensity experiments with $266 \mathrm{~nm} / 36$ ps pulses and $248 \mathrm{~nm} / 12$ ns pulses: dimerization (cf. II in Figure 1) and formation of maleic acid (cf. III in Figure 1).

Based on qualitative estimates, these authors assign the dimerization to a higher excited triplet state $T_{k}$ and the water addition reaction to a higher excited singlet $S_{n}$ (see also Figure 1).

Because of the pioneering character of these maleic acid investigations for nonlinear laser chemistry ${ }^{2}$ a quantitative understanding is highly desirable.

The first problems to be solved in this direction are the determination of population distribution in the excited electronic states at the given excitation conditions, connected with a characterization of the actual maleic acid energy level scheme involved in the excitation and deactivation processes.

Related practical questions are the optimization of the laser pulse parameters (excitation conditions) to get a selective excitation of e.g. $T_{k}$ or $S_{n}$ as high as possible. Prerequisite of this quantitative handling is the knowledge of all processes (and of

† Karl-Weierstraß-Institute of Mathematics, Mohrenstraße, GDR-1986, Berlin.

¥ Central Institute of Physical Chemistry, Rudower Chaussee, GDR-1199, Berlin. 

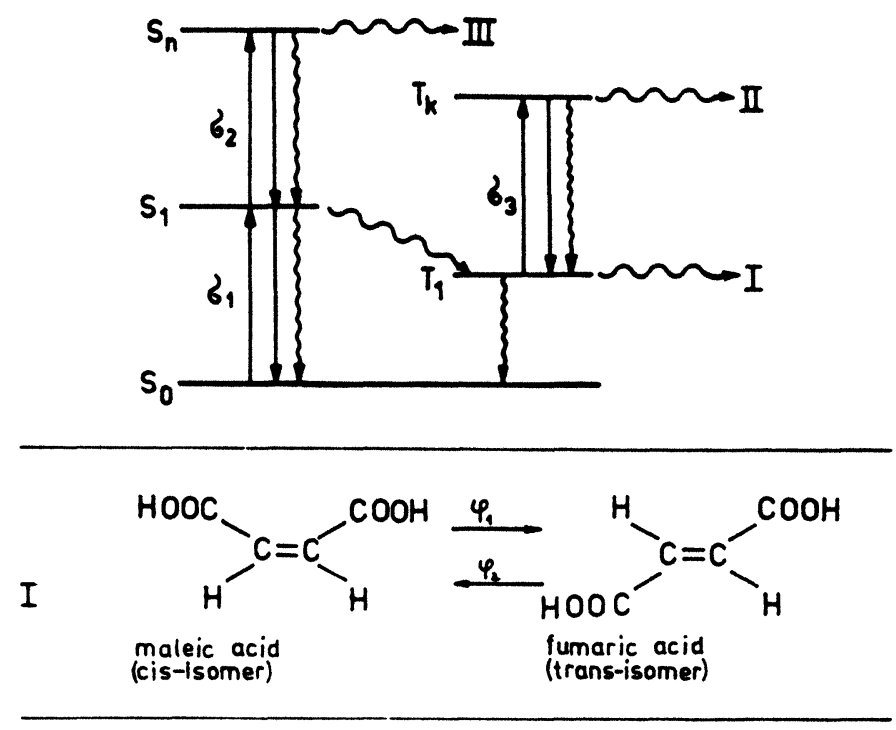

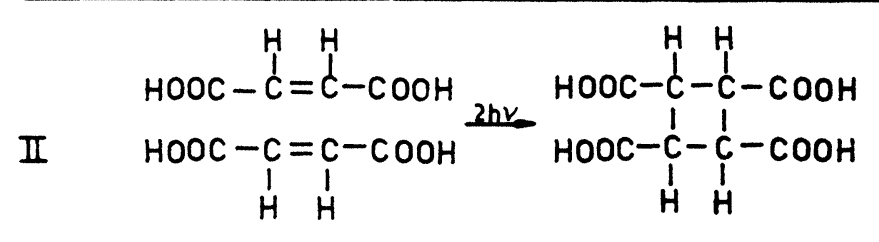<smiles></smiles>

Figure 1 Part of the energy level scheme of the maleic acid molecule in aqueous solution, with indication of excited state reactions (according to Ref. 1-4).

their constants) in the excited states involved in the pumping and deactivation process. This knowledge is, however, very restricted. With the aqueous solution of maleic acid the first absorption of the $248 \mathrm{~nm}$ or $266 \mathrm{~nm}$ photons takes place within the $S_{0}-S_{1}$ transition with cross sections of $\sigma_{1}(248)=4.6 \cdot 10^{-18}$ and $\sigma_{1}(266)=$ $1.9 \cdot 10^{-18}$.

Besides the quantum yield of isomerization, $\phi_{1}=0.04^{6}$, these are the only known molecular constants for the manifold of excited state processes indicated in the term scheme of Figure 1. Such restricted knowledge is not a characteristic of maleic acid, but this is a typical starting situation for laser chemistry in general.

In the following a Physico-Mathematical Methods Package of Nonlinear Absorption/Emission is presented, which was developed to overcome these said difficulties and to obtain the basis for a quantitative excited-state spectroscopy and 
chemistry of organic molecules. The application of this Methods Package to maleic acid results in the determination or at least order-of-magnitude estimation of eight hitherto unknown excited state parameters and leads to the conclusion that assignment of the maleic acid photoproducts to origin in $S_{n}$ and $T_{k}$ should be revised.

\section{THE PHYSICO-MATHEMATICAL METHODS PACKAGE OF NONLINEAR ABSORPTION/EMISSION}

This package of methods is applicable to those cases of practical interest where the excited state population of organic molecules is realized by means of nano or picosecond laser pulses, i.e. to such radiation-matter interactions which can be described by the coupled system of differential equations for photon transport and rate equations.

The basic idea is to take the information missing with respect to the molecular constants of excited states (e.g. absorption cross sections and relaxation constants) and the actual part of the involved energy level scheme from the interaction process itself. Experimentally accessible quantities which implicitly contain this information are the nonlinear absorption (transmission measurement using the pulse that realizes the excited state population, or using a simultaneous or delayed test pulse) and the nonlinear emission (e.g. in the form of intensity dependence of the luminescence quantum yield in the Stokes or in the anti-Stokes range).

These measurements of nonlinear absorption and/or emission are the first part of the package of methods. The implicit information obtained by these measurements is in the second part transformed into explicit statements, e.g. concerning the minimum range and the structure of the involved level scheme and boundaries for unknown excited state constants. This second part is based on analytical investigations of the above-mentioned system of differential equations for general model schemes of energy levels. They concern mainly the behaviour of the quantities of interest (e.g. transmission, population densities, fluorescence quantum yield) at the limits of pumping intensity and time as well as for asymptotic approximation to these limits. The main part of this information is available in the form of an expert system, realized as a dialog with a microcomputer.

The knowledge obtained in this way forms the starting information for the numerical treatment of the problem to be carried out in the third part of the package.

Using the numerical package (realized by ESER 1055 software as well as 16-bit microcomputer software), the nonlinear quantities (functions) which were measured are calculated and compared first of all with the experimental curves. Then, using minimization methods, the still unknown excited-state parameters are varied until an optimum agreement between the calculated and the measured curves is achieved. For this set of optimum parameters the population density of all energy levels involved is then calculated as a function of intensity, time and position in the sample.

Special care is taken.for the uniqueness of this optimum set by mathematical as well as physical procedures (e.g. analysis of sensitivity, variation of pulse duration). If a certain population density distribution is desired in practice (e.g. an enrichment 
to high percentages at a special level), then, on the basis of the set of optimum parameters, the possibilities of realizing this aim can be evaluated by mathematically simulating a variation of the excitation conditions. The complete hardware and software of all three parts of the Physico-Mathematical Methods Package of Nonlinear Absorption/Emission is combined to a uniform set-up (LIS 302).

\section{APPLICATION TO MALEIC ACID}

In the following the population density distribution in the level scheme according to Figure 1 for the experimental pumping conditions of Letokhov et al. (Photonflux density $\simeq 10^{26} \mathrm{~cm}^{-2} \mathrm{~s}^{-1}$ with the $248 \mathrm{~nm} / 12 \mathrm{~ns}$ pulses and between $1.45 \cdot 10^{26}$ and $8 \cdot 10^{27} \mathrm{~cm}^{-2} \mathrm{~s}^{-1}$ with the $266 \mathrm{~nm} / 36 \mathrm{ps}$ pulses) are calculated as a first approach to the desired complete understanding of the maleic acid excited state processes.

Prerequisite for this is the determination (or at least order-of-magnitude estimation) of the following seven unknown parameters (cf. Figure 1): $\sigma_{2}, \sigma_{3}, k_{S_{1} s_{0}}, k_{S_{1} T_{1}}$, $k_{S_{n} S_{1}}, k_{T_{k} T_{1}}, k_{T_{1} S_{0}}$.

To allow a comparison with the known value of steady-state quantum yield of isomerization, ${ }^{6}$ the order of magnitude of $k_{\text {iso }}$ is also estimated. The problem is solved within the framework of the Physico-Mathematical Methods Package described above.

The transmission measurements under (extended) pumping conditions were used as implicit information:

a. Transmission of an aqueous solution of maleic acid for the $266 \mathrm{~nm} / 36 \mathrm{ps}$ pulses with photonflux densities between $10^{26} \mathrm{~cm}$ and $3 \cdot 10^{28} \mathrm{~cm}^{-2} \mathrm{~s}^{-1}$. As shown by Noginova and Matveet $z^{7}$ the energy-transmission is constant over the entire measuring range and corresponds to the low-signal value $(T=0.18, d=0.1 \mathrm{~cm})$. In the following we refer to this result as the "ps case."

b. Transmission of an aqueous solution of maleic acid for the $248 \mathrm{~nm} / 13 \mathrm{~ns}$ pulses with photonflux densities between $10^{24}$ and $3 \cdot 10^{26} \mathrm{~cm}^{-2} \mathrm{~s}^{-1}$. As shown in Figure 2 the peak power transmission decreases. The experimental set-up used is described in the Appendix. In the following we refer to this result as the "ns case."

Taking these experimental results as the start information for part two of the Methods Package the analytical treatment yields firstly the fact that the five level system with three radiative transitions according to Figure 1 is sufficient to describe both transmission cases simultaneously. Moreover, the expert system yields general relations connecting all excited state parameters (except $k_{S_{n} s_{1}}$ and $k_{T_{k} T_{1}}$ ) and special sets of inequalities for the excited state cross sections at both the ns and the ps case. Based on this, preliminary values for all the seven necessary but hitherto unknown excited state constants were chosen from which the start set for the numerics (the third part of the Methods Package) was prepared. Because the simulation of the experimental results on the basis of this start set was not perfect naturally, minimization procedures were activated and the sensitivity of transmission behaviour 


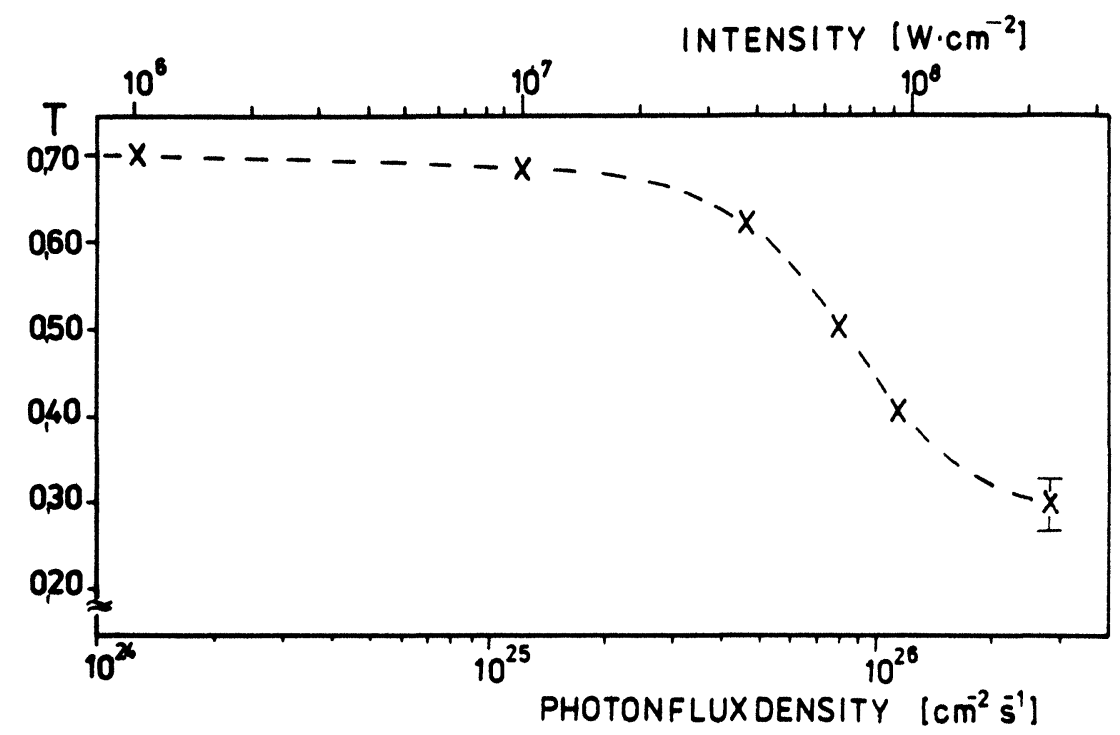

Figure 2 Nonlinear absorption (intensity dependence of peak power transmission) of an aqueous maleic acid solution at $\lambda=248 \mathrm{~nm}$. Thickness of sample: $0.1 \mathrm{~cm}, T_{0}=71 \%$, pulse duration (fwhm): $13 \mathrm{~ns}$.

against variation of all unknown excited state cross sections and relaxation constants was studied.

For the ps case it resulted in maximum sensitivity for $k_{S_{1} S_{0}}, k_{S_{1} T_{1}}$ and $k_{S_{n_{0}} s_{1}}$. Especially $k_{S_{1} T_{1}}$ is well fixed by the experimental data to a value of $k_{S_{1} T_{1}} \simeq 4 \cdot 10^{10} \mathrm{~s}^{-1}$, whereas $k_{S_{1} S_{0}}$ and $k_{S_{n} S_{1}}$ are connected by the relation $k_{S_{n} S_{1}} \simeq 4 k_{S_{1} S_{0}}$ with a nearly one order-of-magnitude uncertainty of the absolute values.

This can be eliminated by taking into account the ns case, which moreover allows to estimate $\sigma_{3}$ and to obtain information on the sensitivity for the other parameters. In this way the following optimum set of excited state constants was found, which agrees with the known value of quantum yield of fumaric acid formation:

$$
\begin{aligned}
\sigma_{2} & =\sigma_{1}, \sigma_{3}(248 \mathrm{~nm})=1 \cdot 10^{-16} \mathrm{~cm}^{2} \\
k_{S_{1} S_{0}} & =9 \cdot 10^{11} \mathrm{~s}^{-1}, k_{S_{1} T_{1}}=4 \cdot 10^{10} \mathrm{~s}^{-1} \\
k_{S_{n} S_{1}} & =4 \cdot 10^{12} \mathrm{~s}^{-1} \\
k_{T_{k} T_{1}} & \simeq 10^{11} \mathrm{~s}^{-1}, k_{T_{1} S_{0}} \simeq 10^{7} \mathrm{~s}^{-1}, k_{\text {iso }} \simeq 10^{8} \mathrm{~s}^{-1}
\end{aligned}
$$

\section{COMPARISON BETWEEN CALCULATED EXCITED STATE DATA AND PUBLISHED PHOTOCHEMICAL RESULTS}

The population densities of all relevant energy levels of the maleic acid term scheme can be calculated for the pumping conditions in the photochemical experiments of Letokhov et al. with the optimum set of excited state constants. The quantum yield of selected pathways can also be determined. As an example, in Figure 3 the peak 


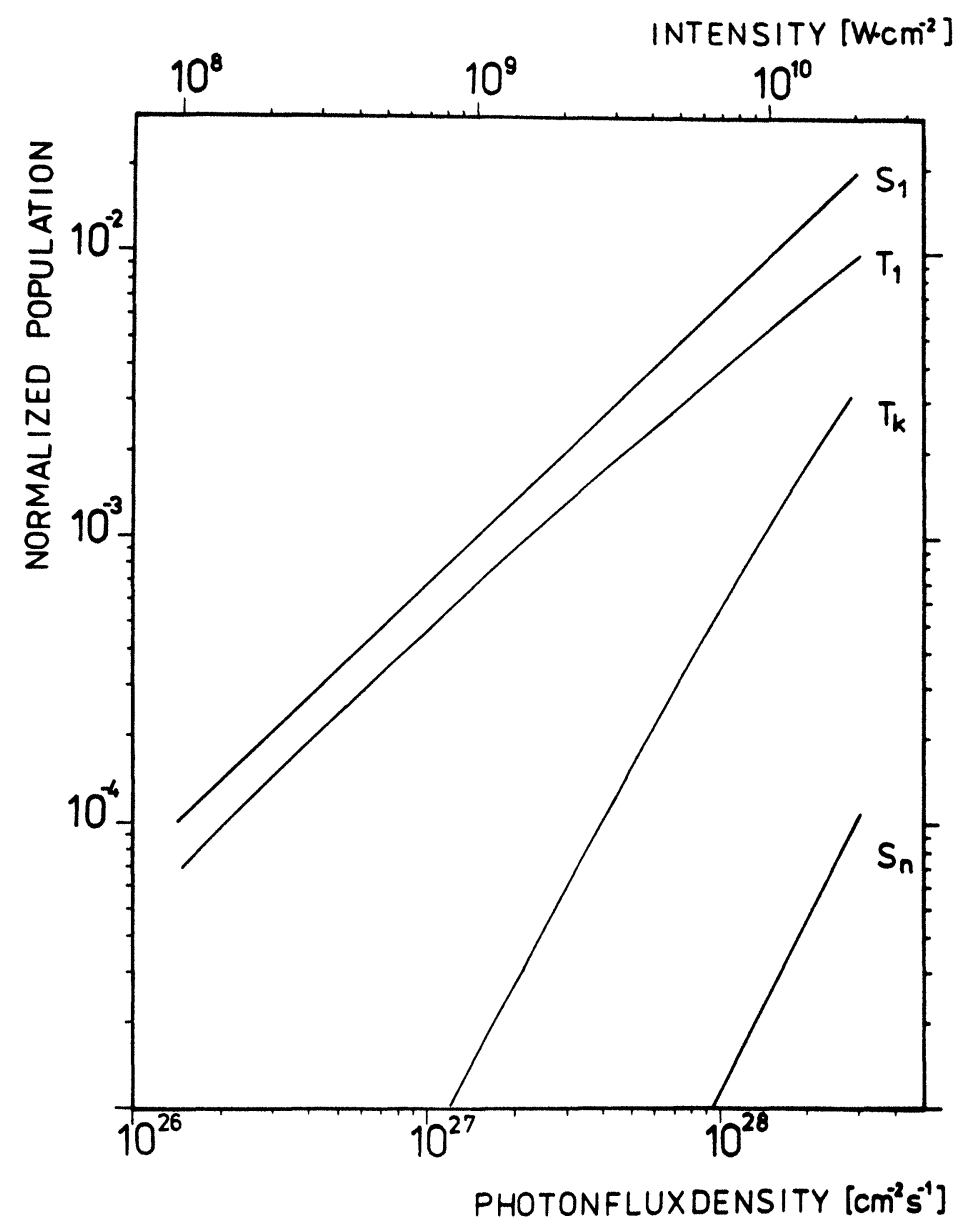

Figure 3 Normalized population densities of the levels $S_{1}, S_{n}, T_{1}, T_{k}$ at pulse maximum with psexcitation between $10^{8}$ and $2 \cdot 10^{10} \mathrm{~W} \mathrm{~cm}^{-2}$ (pulse duration (fwhm) : $30 \mathrm{ps}$ ).

population of the levels $S_{1}, S_{n}, T_{1}$ and $T_{k}$ at ps-excitation with photon-flux densities between $1.4 \cdot 10^{26}$ and $3 \cdot 10^{28} \mathrm{~cm}^{-2} \mathrm{~s}^{-1}\left(10^{8} \div 2 \cdot 10^{10} \mathrm{~W} \mathrm{~cm} \mathrm{~cm}^{-2}\right)$ are shown. (Especially for the exit layer of the small-thickness 35\%-transmission sample of Letokhov's experiments.)

From this figure it is noteworthy that the $S_{n}$-population with the described experimental condition was extremely low; therefore first doubts arise whether $S_{n}$ is the origin of the water addition reaction with maleic acid formation ${ }^{1-4}$ (cf. Figure 1).

Whereas the experimentally determined quantum yield of this maleic acid reaction $^{4}$ is a raising convex-shaped function of intensity with indication of saturation at about $10^{10} \mathrm{~W} \mathrm{~cm}^{-2}$, the corresponding calculated curve (not shown) is strongly raising, concave-shaped and far from any saturation.

There are also differences between calculated behaviour and interpretation of 
experimental results concerning the proposed dimerization at $T_{k}$. Whereas the dimer quantum yield decreases with increasing ps-pump power, ${ }^{1}$ from the calculations an increase would be expected. The only level which in the framework of the present model could be responsible for a quantum yield decrease with increasing power is $T_{1}$ (see below).

Therefore we propose as a hypothesis the following change in designation of the reaction pathways for the laser produced products of maleic acid.

1. The malic acid formation takes place in $T_{k}$ (not in $S_{n}$ ). In a slightly extended model we have simulated this reaction by coupling a new level $X$ to $T_{k}$ (without further new processes in the ps time scale). The calculated intensity dependence of quantum yield of $X$-formation for the ps-case is shown in Figure 4, a slight indication of beginning saturation can be seen. (A more perfect simulation of the experimental result ${ }^{4}$ could not be expected with that model because of the following.)

2. The dimer formation takes place between two maleic acid molecules each in $T_{1}$, which are loosely coupled together already in the ground state (e.g. by $\mathrm{H}$ bridge $^{4}$ ).

Whereas in the framework of the original model (cf. Figure 1) the $T_{1}$-yield for the ps-case shows already a slight decrease with increasing pump power (cf. Figure 4),

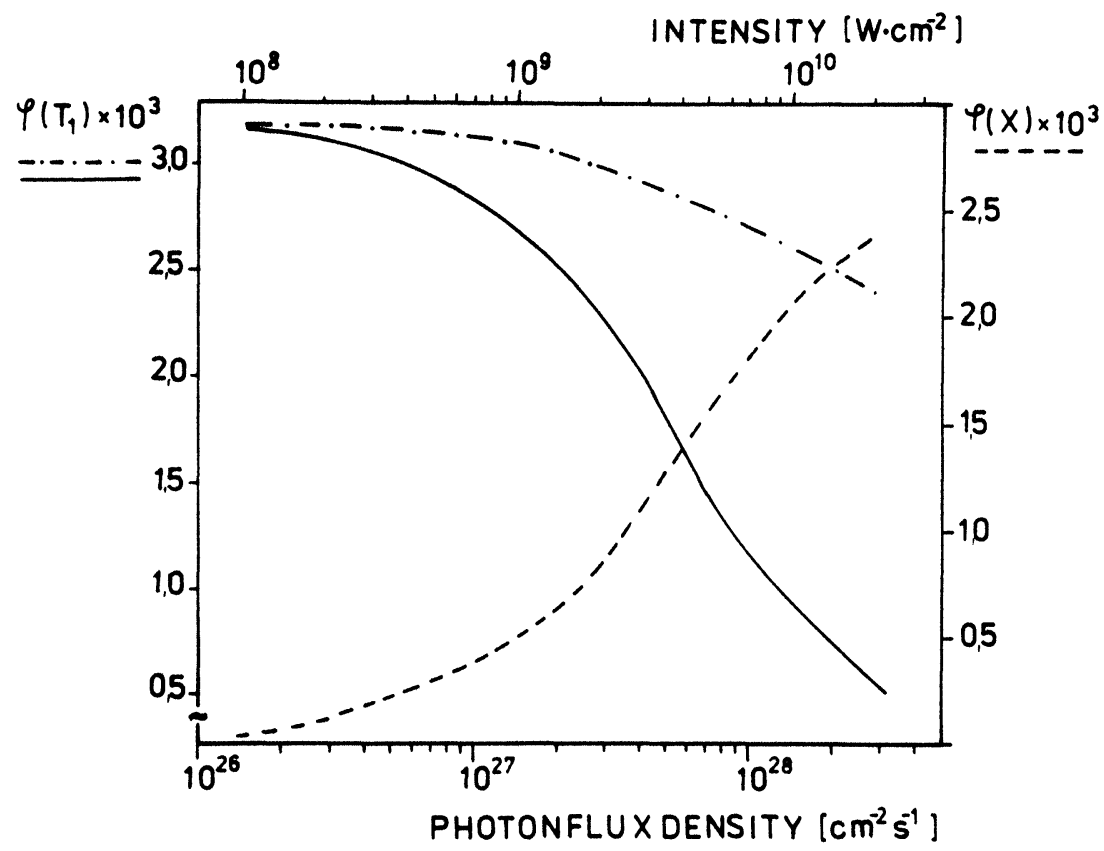

Figure 4 Intensity dependence of $T_{1}$-yield -.-.- (original level scheme of Figure 1) and of $T_{1}$-yield and $X$-yield - - - for an extended level scheme (cf. text) with ps-excitation. 
this effect is enhanced due to the assumed water addition reaction in $T_{k}$ (channel $X$, see above), which is also shown in Figure 4.

We expect that on this basis the explicit inclusion of a $T_{1}+T_{1} \rightarrow$ dimer reaction in the calculations will give a reproduction of the experimental result of dimer quantum yield decrease with increasing ps-pump power. Related work is in progress.

\section{References}

1. E. V. Khoroshilova, N. P. Kuzmina, V. S. Letokhov and Yu. A. Matveetz, Appl. Phys. B31, 145 (1983).

2. V. S. Letokhov (ed.) in Nonlinear Laser Chemistry, Springer Series in Chemical Physics 22 (Springer, Berlin, Heidelberg, New York, 1983).

3. E. V. Khoroshilova, N. P. Kuzmina, V. S. Letokhov and Yu. A. Matveetz, in Photochemistry and Photobiology, edited by A. H. Zewail (Haarwood, Chur, 1983) Vol. 2; p. 1267.

4. Yu. A. Matveetz and E. V. Khoroshilova, in Laser ps-Spectroscopy and Photochemistry of Biomolecules, edited by V. S. Letokhov (A. Hilger, London, 1987) p. 283.

5. G. Porter, Angew. Chemie 80, 882 (1968).

6. A. R. Olson and F. L. Hudson, J. Am. Chem. Soc. 55, 1410 (1933).

7. N. E. Noginova, work for diploma, sc. leader Yu. A. Matveetz, Troitzk, 1984.

\section{APPENDIX}

\section{Experimental Set-Up for Nonlinear Absorption Measurement}

A self-made (CIPC) $\mathrm{KrF}$ laser was used with pulse energy of $8 \mathrm{~mJ}$ and pulse duration of $13 \mathrm{~ns}(\mathrm{fwhm})$ as the light source.

The radiation was focused by a $150 \mathrm{~mm}$ lens to the $1 \mathrm{~mm}$ sample cell. Variation of intensity was realized by calibrated filter sets. Each laser shot was directed onto a formerly unexcited sample volume. The transmitted radiation was directed via an $80 \mathrm{~mm}$ lens to a pyroelectric detector (type J3-05, Molectron).

To measure the peak power of transmitted radiation, this detector was connected with a $100 \mathrm{MHz}$ oscillograph (type $1555 \mathrm{EMG}$ ). 